\title{
Sleep duration is associated with daily consumption of fruits and vegetables in British women from The UK Women's Cohort Study
}

\author{
E.A. Noorwali ${ }^{1,2}$, J.E. Cade ${ }^{1}$, V.J. Burley ${ }^{1}$ and L.J. Hardie ${ }^{3}$ \\ ${ }^{1}$ Nutritional Epidemiology Group, School of Food Science and Nutrition, University of Leeds, Leeds LS2 9JT, UK, \\ ${ }^{2}$ Department of Clinical Nutrition, Umm Al-Qura University, Saudi Arabia and \\ ${ }^{3}$ Division of Epidemiology and Biostatistics, School of Medicine, University of Leeds, Leeds, LS2 9JT, UK
}

The World Health Organization recommends consuming at least 400 grams of fruits and vegetables (FV) per day to improve overall health and reduce the risk of chronic diseases, however, FV consumption remains below the recommended levels in the UK ${ }^{(1,2)}$.There are limited studies assessing the association between sleep duration and FV consumption in adults ${ }^{(3)}$. The present study aims to explore the association between sleep duration and FV intakes in middle aged British women from the UK Women's Cohort study (UKWCS).

Cross-sectional and prospective data were obtained from the UKWCS. Sleep duration, the exposure, was assessed by self-report of average hours slept on weekdays and weekends. Dietary intake, as an outcome, was assessed by a 4-day food diary cross-sectionally and in a follow-up study 4-5 years later, which was used for the prospective analysis. In addition, participants were asked in a health and lifestyle questionnaire "How many servings of fruit/vegetables or dishes containing fruit/vegetables do you usually eat in an average week. 2253 women were followed-up and 451 fully-coded food diaries were included in the prospective analysis. Linear regression analysis, adjusted for confounders, was used to assess the relationship between sleep duration and FV intakes."

13,652 women were included in the cross-sectional analysis with a mean age 52 years (95\%CI 52, 53). Sleep duration was divided to 3 categories: short $(<7 \mathrm{~h})$, recommended $(7-8 \mathrm{~h})$ and long $(>8 \mathrm{~h})$. In adjusted models, cross-sectional analysis showed that long sleepers (LS) consumed less FV than short sleepers (SS) and recommended sleepers (RS). LS consumed on average 20 grams/day $(95 \% \mathrm{CI}-29,-10)$ less total FV in comparison to RS $(\mathrm{P}<0 \cdot 001)$. Prospectively, LS consumed $1 \cdot 7(95 \% \mathrm{CI}-3,-0 \cdot 27)$ less portions of total FV/week $(\mathrm{P}=0.02)$ compared to RS (Table 1.). The relationship between sleep duration and FV intake was non-linear $(\mathrm{P}<0.001)$ with women sleeping the recommended duration of $7-8 \mathrm{~h}$ having the highest intakes compared to SS and LS.

Table 1. The association between sleep duration categories and FV intakes prospectively of women aged 33-73 years from the UK women cohort study.

Sleep categories compared to Recommended sleepers (7-8hours)- reference category

\begin{tabular}{|c|c|c|c|c|c|c|}
\hline \multirow[b]{3}{*}{ Model* } & \multicolumn{3}{|l|}{ Short sleepers } & \multicolumn{3}{|l|}{ Long sleepers } \\
\hline & \multicolumn{3}{|l|}{$<7$ hours } & \multicolumn{3}{|l|}{$>8$ hours } \\
\hline & Difference of means & $95 \% \mathrm{CI}$ & $P$ value & Difference of means & $95 \% \mathrm{CI}$ & $\mathrm{P}$ value \\
\hline Fruit $(\mathrm{g} / \mathrm{d})^{* *}$ & -29 & $-66,6$ & $0 \cdot 1$ & -28 & $-61,3$ & $0 \cdot 08$ \\
\hline Vegetables $(\mathrm{g} / \mathrm{d})^{* *}$ & -23 & $-50,4$ & 0.09 & -7 & $-31,16$ & $0 \cdot 5$ \\
\hline Total FV $(\mathrm{g} / \mathrm{d})^{* *}$ & -50 & $-101,0 \cdot 38$ & 0.05 & -37 & $-82,7$ & $0 \cdot 1$ \\
\hline Fruit (serving/week)*** & $-0 \cdot 29$ & $-1,0.72$ & $0 \cdot 5$ & -0.93 & $-1,-0 \cdot 02$ & 0.04 \\
\hline Vegetables(serving/week) ${ }^{* * *}$ & 0.42 & $-0 \cdot 66,1$ & $0 \cdot 4$ & -0.73 & $-1,0 \cdot 23$ & $0 \cdot 1$ \\
\hline Total FV(portion/week)*** & $0 \cdot 11$ & $-1,1$ & $0 \cdot 8$ & $-1 \cdot 7$ & $-3,-0 \cdot 27$ & 0.02 \\
\hline
\end{tabular}

$\mathrm{G}$, gram, d, day, FV, fruits and vegetables, CI, Confidence interval, * adjusted for age, socio-economic status \& smoking, **n $=451$ obtained from 4-day food diary $* * * n=2253$ obtained from health and lifestyle questionnaire.

The results of this study suggest a link between sleep duration and FV consumption. These findings may have important implications for lifestyle and behaviour change policy. Future research should incorporate objective measures of sleep in intervention trials to determine whether sleep duration has a direct impact upon FV intake.

Essra Noorwali is in receipt of a scholarship from Umm Al-Qura University, Makkah, Saudi Arabia.

1. World Health Organization (2003) report of a Joint WHO/FAO expert consultation, 149.

2. Aune D, Giovannucci E, Boffetta P et al. (2017) Int J Epidemiol, 1-28.

3. Dashti HS, Scheer FA, Jacques PF et al. (2015) Advances in Nutrition 6, 648-659. 